\title{
Modelling Spatial and Temporal Agent Travel Patterns for Optimal Charging of Electric Vehicles in Low Carbon Networks
}

\author{
Salvador Acha, Member, IEEE, Koen H. van Dam, Member, IEEE, and Nilay Shah
}

\begin{abstract}
The ability to determine optimal charging profiles of electric vehicles (EVs) is paramount in developing an efficient and reliable smart-grid. However, so far the level of analysis proposed to address this issue lacks combined spatial and temporal elements, thus making mobility a key challenge to address for a proper representation of this problem. This paper details the principles applied to represent optimal charging of EVs by employing an agent-based model that simulates the travelling patterns of vehicles on a road network. The output data is used as a reliable forecast so an optimal power flow model can devise optimal charging scenarios of EVs in a local electrical network. The effectiveness of the model is illustrated by presenting a multi-day case study in an urban area. Results show a high level of detail and variability in EV charging when a present-day carbon fuel mix is compared to one with lower carbon intensity.
\end{abstract}

Index Terms-Agent based transport modelling, coordination of distributed energy resources, distribution network operation, electric vehicle charging, local smart-grids, optimal power flow.

\section{INTRODUCTION}

$\mathrm{E}^{\mathrm{s}}$ LECTRIC vehicle technology represents a challenging and stimulating task in power system engineering since its intrinsic mobility characteristic means loads can appear and disappear in different parts of the network. Addressing this issue will require thorough research in driving patterns and vehicle usage; thus providing utilities the capacity to predict when and where these vehicles may require energy for their travelling needs while also guaranteeing they are capable of supplying this new stochastic demand whenever required.

Using modelling and simulation power system engineers approach key issues concerning optimal design and operation analysis of distribution networks [1], [2]. With the introduction of EVs and other distributed energy resources (DERs), researchers of distribution networks need to re-think some of the assumptions that have become common place in literature since networks will undoubtedly become pro-active in order to adapt to ever-changing conditions and hence its models should accommodate these features [3].

S. Acha is with the Department of Chemical Engineering, Imperial College, London, UK SW7 2AZ (e-mail: salvador.acha@ic.ac.uk).

K.H. van Dam is with the Department of Chemical Engineering, Imperial College, London, UK SW7 2AZ (e-mail: k.van-dam@ic.ac.uk).

N. Shah is with the Department of Chemical Engineering, Imperial College, London, UK SW7 2AZ (e-mail: n.shah@ic.ac.uk).
The IEA Technology Roadmap on EVs and plug-in hybrid EVs suggests that by the year 2050 sales for 100 million of these vehicles is achievable [4] and car manufacturers such as Nissan, Tesla and BMW are launching models for residential and commercial customers [5]. With a growing market penetration, the case to determine optimal charging profiles of EVs in local electricity distribution networks is paramount in developing an effective and robust smart-grid.

This paper proposes a holistic framework so power system engineers can represent and determine - in a reliable manner - the optimal charging of EVs and the related electricity flows in distribution networks. The model proposed is conformed from a two-step solution. Firstly, an agent-based model (ABM) portrays travel patterns of EV users in an urban area respecting the profile of drivers and consequently quantifying their spatial, temporal, and energy demand. The output data from the ABM helps a distribution network operator (DNO) to forecast energy demands of EVs. Secondly, this data is applied to an optimal power flow (OPF) model to resolve ideal EV charging times using a multi-objective cost function. A case study is presented showcasing the granular features the framework offers to decide when and where EVs are charged in an urban area with a local electric network. The modelling approach confirms it is possible for stakeholders to begin assessing with a high level of detail the impact EVs can have on networks when optimal charging is desired.

\section{Modelling Electric Vehicle Charging And Mobility}

Optimal charging of EVs is an intriguing and relevant topic within the smart-grid theme. The problem itself is susceptible to many factors that can influence results to varying degrees. These factors could include flexible electricity tariffs, carbon content of the power used to fuel the EV, battery capacity, rate of charge characteristics, vehicle travel patterns and user activities and network features (e.g. topologies) just to mention a few. Therefore, for power system engineers, optimal EV charging is a novel field of research with unresolved issues from a technical-economical, market, and policy perspective.

Research summaries on measuring and modelling the impact of EVs on the distribution grid and a literature study on the management and aggregation of EV charging have been presented in [6] and [7]. Thus far a key challenge when tackling this peculiar problem is the fact that researchers have 
to make broad assumptions when addressing mobility issues in power flow calculations [8]. Data on vehicle usage and resulting energy demands are generally based on aggregate data; for example composed of census data and average trip distances [9] or by combining local travel surveys with comparable datasets from other countries [10]. This data is then translated into load curves for EVs that can be added to existing load profiles. In other words, there is still a gap between modelling travel patterns of vehicles and combining them with conventional power flow analysis.

Because there is limited data available on travel surveys for light duty vehicles most EV studies in power systems literature show a lack of granular depth within local networks. Furthermore, work based on survey data does not detail how EVs move through the networks and hence have been limited by assuming vehicles obey a specific travel pattern. These papers usually aim at either improving network performance [9] or reducing charging costs to EV owners while also making use of low carbon electricity [10]. Another recurrent topic in the literature is EV capability in demand response strategies via third party aggregators due to the load flexibility benefits battery storage in EVs bring to power systems [11].

In the above cases limited spatial data is generally sufficient, but when studying the effects of EVs on the distribution grid more detailed data is required. An alternative is using an activity-based approach to trip-generation which results in spatial-temporal information on $\mathrm{EV}$ movements [14]. Such activities can then be translated into energy requirements with the advantage that the spatial dimension is also known. In a similar fashion, [15] uses micro-simulation of traffic to create vehicle profiles which are then used to study the impact of EVs on the electricity network in the city of Zurich [16]. These approaches use the so called agentbased modelling paradigm, which is a bottom-up way to model actors in a system, including their autonomous actions and interactions.

Given the socio-technical interactions and the distributed nature of electricity systems, in particular when including DERs, it is no surprise that this paradigm has been widely used to study EV and smart-grid phenomena with results published in artificial intelligence or autonomous agents literature in addition to addressing the power systems field. For example, the acceptance of EVs as an alternative to an internal combustion car has been studied with $\mathrm{ABM}$ in which the decision of households to buy a new car is central [17], [18]. Furthermore, an agent-based market model has been developed to match supply and demand of DERs [19] and has been applied in a living lab enabling optimisation of EV charging using actual hardware [20].

Agents have also been used for management of electricity storage, including EV batteries, as a way to minimise peak loads [21]. Similarly, an ABM framework is used to connect various models of the energy infrastructure including demand and supply models complemented with traffic simulation which is then used to study the effects of vehicle-to-grid

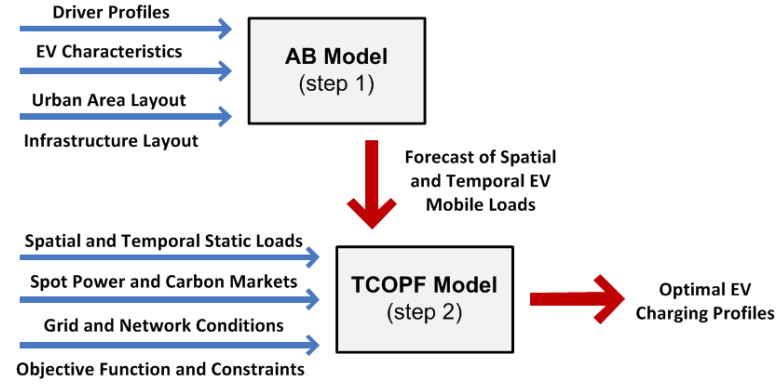

Fig.1 - Integration of agent-based and optimal power flow models to analyse the effects of electric vehicles on an electricity network.

(V2G) for balancing wind energy [22]. Meanwhile, [23] proposes an $\mathrm{ABM}$ approach for charging EVs linked to imbalance reduction with a model in which vehicle agents, transformer agents and balancing responsible parties coordinate their tasks for charging, operating the network, and supplying electricity, respectively. The bottom-up approach of ABM thus offers a new perspective on power systems modelling in which the activities of various actors, including owners of EVs and utility companies are modelled to gain new insights in the management of power systems.

\section{Integrating An Agent-BAsed EV Model AND AN OPTIMAL POWER FLOW MODEL}

As the literature review suggests, in order to gain the most from the deployment of EVs it is imperative that power system engineers address the challenges these units bring to utilities with their mobility feature as the most important one. Hence, understanding how to best represent EV driving profiles is fundamental. This work continues an encouraging approach to represent EV agents in optimal power flow studies through a holistic framework using both $\mathrm{ABM}$ and TCOPF [8].

Figure 1 illustrates how the two models are connected and what type of data inputs are required. A feature of the ABM consists in keeping track of the energy consumption EVs have at each time interval as well as their location in the network. This valuable temporal and spatial data provided by the ABM is then employed by the TCOPF; effectively functioning as an intermediary entity making it possible to assess optimal EV charging profiles and their impact on distribution networks.

The two models are used to merge transport and electrical systems into a single analytical framework. This way the bottom-up ABM perspective, representing vehicle owners and their travel behaviour, can be combined with the top-down view from the DNO which can overview the power network and therefore optimise EV charging over time at system level.

\section{A. Agent-based Model of Electrical Vehicle Usage}

The ABM describes the behaviour of EV owners, modelled as autonomous agents, and their activities bring them to various part of the city at different times of the day. These activities range between work and leisure. The model keeps track of the position of each vehicle for each time interval as well as the state of charge (SOC) in the battery which is 
discharged as a result from travelling.

The first input of the ABM consists of driver profiles of EV owners which populate an urban area under study. Each driver has a number of characteristics, such as home location, its work place (if applicable) and number of children (if any). While a limited number of profiles has been pre-defined, the model can clone these agents to populate an area with any number of EV owners. Additionally, the characteristics of the fleet of EVs used in the model are given as an input; including the battery capacity and travel efficiency in $\mathrm{kWh} /$ miles.

Furthermore, the model requires a city layout as input. This data is read from GIS shape files with the coordinates of buildings, roads, and electrical network. The model uses the layout to determine journeys of the agents based on their activities as well as their location for when an EV is plugged.

Activities performed by agents in the model can vary from one another depending on their particular profiles as well as the time of day and the day of the week being assessed. For instance, those with a job drive to their work place but if they have kids they drop them off at school first. Meanwhile, other activities can be planned around these core tasks. Random distributions are employed to determine individual departure times as well as type and location of the next activity.

An assumption in the model is that when an agent is not driving its EV is defined to be plugged into the network. The location of the nearest substation is determined based on where the EV is parked. At each interval, all EVs plugged are aggregated per node and this allows the model to determine maximum rate of charge and the SOC of the batteries. As a result, the output data gives spatial and temporal EV fleet load flexibility per node. Note that in the ABM model the battery of EVs is not charged as it is initialised with a full charge.

The ABM has been implemented in Repast Simphony building on top of the open-source Repast City model [24]. Existing micro-simulation traffic models and other agentbased models of driver behaviour may be more advanced and provide more realistic traffic simulation (e.g. including congestion or traffic light control), but instead a relatively simple model is used which focuses on the challenges mentioned above. The simulation can be run for one or multiple days and it produces activity logs for the individual agents and statistics on total distances driven and energy consumed (for each EV as well as for the entire population) in addition to the results on the load flexibility. The outputs are then fed into the OPF model for optimisation of EV charging.

\section{B. Electric Vehicle Optimal Power Flow}

In this paper a simplified version of the time coordinated optimal power flow (TCOPF) presented in [25] is used to incorporate the data provided by the ABM to resolve EV unit dispatch so these can have an ideal integration to the grid. For a daily load profile the solver analyses the state of the electrical network in its multiple nodes as well as the energy required by the fleet of EVs. After this data is processed the TCOPF can calculate when it is convenient to command EV charging following the specified objective function.
Accordingly, the TCOPF focuses on minimising a nonlinear objective function over multiple period intervals which are restrained by a set of nonlinear constraints. Based on these characteristics, the TCOPF problem formulation can be categorised as a typical steady-state multi-period nonlinear constrained optimisation problem that possesses continuous and mixed-integer properties, while employing piecewise constant functions to regulate its control variables.

For practical purposes, this innovative power flow problem can be seen as having an interesting and useful application for power system studies. Because it can be anticipated that in the near future EVs are abundant in the grid, engineers need to represent the power and energy capacity these technologies bring to power flow studies. Thus, several terms must be added to each nodal balance equation where EV units are under consideration and this condition will allow power engineers to aggregate the capacity EVs represent [26].

The multi-objective function problem tries to consider the key drivers that look to influence how EV technology can be charged efficiently from day to day. These drivers are best represented in monetary terms as follows [12]:

- Day ahead spot electricity market prices

- Carbon cost from charging with fossil fuel electricity

- Network operating costs for energy delivery

\section{Time Coordinated Optimal Power Flow Formulation}

This section details the TCOPF formulation. The objective function focuses on minimising both energy and emission costs incurred from charging EVs. Total costs are composed from price signals given by spot and carbon markets plus grid delivery costs as seen in Equation 1. The solver is holistic and unbiased, thus giving no preference to any particular party either electric vehicle owner or utility companies. The proposed objective function for the EV charging under cost minimisation can be stated as follows:

$$
\begin{aligned}
& \min E V_{\text {costs }}=\min \left(C_{E V \alpha, \beta}+C_{D N O \alpha, \beta}\right) \\
& \min C_{E V}=\min \sum_{\beta=1}^{n \beta}\left[\sum_{\alpha=1}^{n \alpha} P_{E V \alpha, \beta} \cdot\left(\varepsilon_{P \beta}+\chi_{P \beta}\right)\right] \\
& \min C_{D N O \alpha, \beta}=\min \sum_{\beta=1}^{n \beta}\left[\sum_{\alpha=1}^{n \alpha} a P_{D N O \alpha, \beta}+b P_{D N O \alpha, \beta}^{2}\right]
\end{aligned}
$$

Equation (1) is composed from calculating electricity costs from both spot and carbon markets (2) and the electricity costs derived from energy delivery (3). This function establishes the priority of charging EVs when it is most beneficial to vehicle owners, the grid, and the environment. The constraints of the formulation are responsible for defining the region of feasible solution for the energy system being analysed. The TCOPF constraints can be classified into: snapshot (i.e. for each time interval) and global (i.e. for the entire problem horizon). 
Snapshot constraints are subject to each time period $\beta$

$$
\begin{array}{cl}
P_{G \alpha}-P_{D \alpha}-P_{T \alpha}=0 & \forall \alpha \in n \alpha \\
Q_{G \alpha}-Q_{D \alpha}-Q_{T \alpha}=0 & \forall \alpha \in n \alpha \\
V_{\alpha, \text { min }} \leq V_{\alpha} \leq V_{\alpha, \max } & \forall \alpha \in n \alpha \\
|t|_{\alpha, \text { min }} \leq|t|_{\alpha} \leq|t|_{\alpha, \text { max }} & \forall \alpha \in n \alpha \\
P_{E V \alpha, \min } \leq P_{E V \alpha} \leq P_{E V \alpha, \max } & \forall \alpha \in n \alpha \\
E V_{\alpha}^{s o c} \geq 0 & \forall \alpha \in n \alpha
\end{array}
$$

Global constraints are subject to the day being analysed

$$
\sum_{\beta=1}^{n \beta}\left[\sum_{\alpha=1}^{n \alpha} P_{E V \alpha, \beta} \cdot \frac{h r^{\text {total }}}{n \beta}\right]=\operatorname{Total}_{E V} \quad \forall \alpha \in n \alpha, \forall \beta \in n \beta
$$

Equations (4) and (5) refer, respectively, to the nodal balance for active and reactive power flow conservation that must be met in each network node for each time interval. Equations (6) represents voltage limit at nodes while (7) specifies the allowed range of operation for OLTCs. Equation (8) details the flexible EV demand permitted at each node - a key constraint provided by the ABM simulation. Term (9) states all nodal battery storage systems must have at all times a SOC equal to or greater than zero and (10) specifies refuelling batteries based on ABM predicted travel behaviour.

The TCOPF problem is programmed, executed, and solved by performing a multi-period nonlinear optimisation using the gPROMS $^{\mathrm{TM}}$ software [27]. Once the problem is solved a summary report is provided; describing the following results:

- Time consumed during the optimisation process

- Final value of the objective function

- Values for all variables during each time interval

\section{CASE Study And Results}

Using the two models described in Section III, a case study of a small urban area has been set up. In this section the case and the results of the simulations are presented.

\section{A. Case Description and Assumptions}

The aim of the case study is to demonstrate that through ABM the load flexibility of EVs on electrical networks can be estimated with temporal and spatial features. This data then serves as a reliable forecast to support DNOs in supplying EVs in an optimal manner. The case study is determined by driver profiles, EV features, city layout, electricity demands and $11 \mathrm{kV}$ network characteristics, which are described below.

\section{1) Driver Profiles}

The model currently considers three types of EV owners:

1. People with a job, either at an office or leisure centre, who may or may not have kids of school-going age.

2. People without a job, but who have kids of schoolgoing age.

3. Pensioners and other people who do not have to go to work and/or to a school.

There are 14 agents who are loaded from a GIS file, 10 of

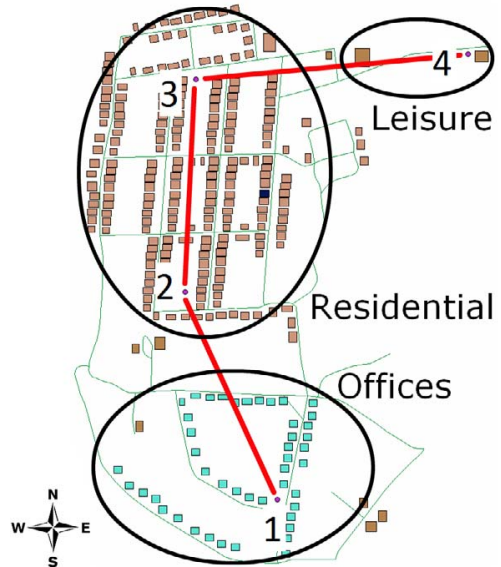

Fig. 2 - Map of the urban area map considered in the case study, with buildings in three different zones as well as road and electrical networks

which are of the first category, 2 in the second and 2 in the third. Based on this distribution 250 agents are generated, each with a different home and work address (if applicable) and a certain number of children. In the model, $80 \%$ of those with a job work in an office and the remaining $20 \%$ in a leisure centre or a shop. In addition, approximately $10 \%$ of the EV owners have to work on Saturday. It is assumed that drivers never deplete their battery in one single day.

\section{2) EV Characteristics}

The EV unit used in the case study is the Nissan Leaf which has a battery capacity of $24 \mathrm{kWh}$ and requires 0.25 $\mathrm{kWh}$ per mile driven, resulting in a driving range of 97 miles on a fully charged battery. A $240 \mathrm{~V}$ and $16 \mathrm{~A}$ charger is considered whenever vehicles are plugged, so the maximum charging rate is $3.84 \mathrm{~kW}$. Characteristics of other EV models can be included, but for simplicity only one vehicle model was used.

\section{3) City Layout}

All geographical data for the city is stored using GIS and Figure 2 shows the city layout used for the case study. The urban area roughly consists of three zones: a central area which predominantly consists of residential premises, the south of the city in which offices are mixed with some leisure areas, and the north-east where leisure centres and shops are concentrated. A road network connects the buildings in the city. Furthermore, the city has four substations that lay the foundations of an $11 \mathrm{kV}$ distribution grid. Two of these power nodes are located in the residential area and one in each of the other two zones. To determine which power node is the nearest to a plugged EV only the geographical distance is considered, so it is possible that a vehicle parked at an office is in fact closest to a node in the residential zone. Thus, there is no strict division between urban areas from the DNOs perspective, yet each node can be characterised by a predominant type.

4) Electricity Load Profiles and Network Characteristics

Besides the demand for electricity to charge EVs resulting from the ABM, load profiles for typical residential and commercial buildings have been used to depict the activities agents perform in their day to day. All load profiles employed 
TABLE I

TCOPF CASE STUDY PARAMETERS

\begin{tabular}{|c|c|}
\hline \multicolumn{2}{|l|}{ Element data } \\
\hline Base Values & Voltage $=11 \mathrm{~kW}$ \\
\hline Electric cables & Admittance $=205.3-j 38.2$ p.u. \\
\hline Slack bus & Voltage $=1 \angle 0^{\circ}$ p.u. \\
\hline Electric vehicles & $\begin{array}{l}\mathrm{PHEV} \text { charge/discharge rate per unit }=3.84 \mathrm{~kW} \\
\text { Battery capacity per EV unit }=24 \mathrm{kWh}\end{array}$ \\
\hline \multicolumn{2}{|l|}{ Constraints } \\
\hline Electric nodes & 0.95 p.u. $\leq V_{\alpha} \leq 1.05$ p.u. \\
\hline Tap changer & $0.95 \leq|t|_{\alpha} \leq 1.05$ \\
\hline \multicolumn{2}{|l|}{ Costs } \\
\hline Scenario 2 & $\begin{array}{l}\mathrm{C}_{\mathrm{DNO}}=10 \mathrm{P}_{\mathrm{DNO}}+0.10 \mathrm{P}_{\mathrm{DNO}}{ }^{2} \\
\text { Carbon price }(\chi)=£ 12 / \text { tonne } \\
\text { Average weekday spot price }(\varepsilon)=£ 73.85 / \mathrm{MWh} \\
\text { Average weekend spot price }(\varepsilon)=£ 64.70 / \mathrm{MWh}\end{array}$ \\
\hline Scenario 3 & $\begin{array}{l}\mathrm{C}_{\mathrm{DNO}}=20 \mathrm{P}_{\mathrm{DNO}}+0.20 \mathrm{P}_{\mathrm{DNO}}{ }^{2} \\
\text { Carbon price }(\chi)=£ 60 / \text { tonne } \\
\text { Average weekday spot price }(\varepsilon)=£ 86.65 / \mathrm{MWh} \\
\text { Average weekend spot price }(\varepsilon)=£ 68.30 / \mathrm{MWh}\end{array}$ \\
\hline
\end{tabular}

Note: Spot prices used change in each time interval and are omitted due to space purposes; however average prices are shown to indicate the tendency that electricity will become a more expensive commodity in the near future. Furthermore, Daily EV energy use is output of the ABM.

are in half-hourly intervals, this input is based on UKGDS data which was adjusted to simulate different customer types [28].

As shown in Figure 2, the $11 \mathrm{kV}$ network has a radial topology and consists of four nodes. It is assumed the system operates under balanced conditions and is represented by its positive sequence network. The load in each node is considered to be a three-phase balanced load. In the south of the city node 1 is located while node 2 and node 3 represent the central area, finally node 4 is in the north-east area.

5) Case Study Scenarios

The case study explores three scenarios. The first does not consider EVs at all serving as a benchmark of the status quo. The second takes the current power generation portfolio in the UK to depict how optimal EV charging would look like within the present context of electricity prices and carbon emissions. The third scenario attempts to look at a future in which renewable sources have a meaningful presence (e.g. $20 \%$ fuel mix). The three scenarios consider $\mathrm{CO}_{2}$ emission variations during the day based on how the fuel mix is composed. This data serves to incentivise when EVs are charged once a cost to carbon is allocated, similar to the work presented in [12]. In all scenarios the same number of EVs is considered and the static as well as mobile demand is identical, however price signals are the only variables that change in each scenario.

The simulation is done for a period of 72 hours, from Thursday morning up to Sunday morning, during which agent activities and demand profiles are suited accordingly to the day of the week. A key assumption from the ABM model is that all batteries have to be fully charged by 6:00 am on weekdays and by 8:00 am on the weekend, thus helping to determine the load flexibility of EVs more effectively. Table I illustrates the energy system parameters used in the TCOPF scenarios.
TABLE II

DISTANCES TRAVELLED PER DAY AND BATTERY STATUS AT END OF DAY

\begin{tabular}{|c|c|c|c|c|}
\hline Day of the week & $\begin{array}{c}\text { Average } \\
\text { (miles) }\end{array}$ & $\begin{array}{c}\text { Minimum } \\
\text { (miles) }\end{array}$ & $\begin{array}{c}\text { Maximum } \\
\text { (miles) }\end{array}$ & $\begin{array}{c}\text { Battery } \\
\text { SOC (\%) }\end{array}$ \\
\hline Thursday & 24.83 & 6.33 & 61.73 & 74.64 \\
\hline Friday & 31.78 & 7.5 & 73.16 & 67.56 \\
\hline Saturday & 23.55 & 3.412 & 66.459 & 75.96 \\
\hline
\end{tabular}

TABLE III

ENERGY USED AND PLUG FACTOR PER DAY

\begin{tabular}{|c|c|c|c|c|}
\hline $\begin{array}{c}\text { Day of the } \\
\text { week }\end{array}$ & $\begin{array}{c}\text { Energy } \\
(\mathbf{M W h})\end{array}$ & $\begin{array}{c}\text { Plug factor } \\
\text { average (\%) }\end{array}$ & $\begin{array}{c}\text { Plug factor } \\
\text { min (\%) }\end{array}$ & $\begin{array}{c}\text { Plug factor } \\
\text { max (\%) }\end{array}$ \\
\hline Thursday & 1.521 & 92.69 & 32.4 & 100 \\
\hline Friday & 1.946 & 90.93, & 35.6, & 100 \\
\hline Saturday & 1.442 & 93.79 & 65.6 & 100 \\
\hline
\end{tabular}

\section{B. Agent-Based Model Results}

The ABM is run once for the three days, after which the same output data is used in second and third scenarios. Table II shows the distance travelled by the agents and the battery status at the end of the day if no charging would take place. There is a large gap between agents who use their EV for only one or two very short trips, and others who use it more frequently. This means that there is a large diversity in travel patterns and thus battery SOCs.

Table III shows the results from the perspective of the aggregator body, featuring the total amount of energy consumed from EV batteries as well as their plug factor (i.e. percentage of units connected to the grid). The plug factor is highly dependent on the time of day, and varies from around $30 \%$ during the morning and evening peak up to $100 \%$ during most of the night. The total amount of energy used over the day is what has to be supplied by the network while the plug factor gives constraints when it might be possible to charge.

Figures 3 to 6 show the energy profiles for each of the four nodes over the course of the three days. MaxSOC denotes the maximum SOC of all batteries in a given node, SOC is the actual charge at that point in time. At the start of the day the SOC and MaxSOC are equal, but over the course of the day this gap widens as electricity of the battery is used. The gap between these two lines gives the load flexibility. A third parameter, LEV, was introduced to give the potential for charging at an interval given the load flexibility as well as the maximum charging rate.

From the figures, typical daily travel patterns can be observed, such as large volume of cars which start to arrive early in the mornings at node 1 , where the offices are based, after having departed from residential nodes 2 and 3. On the weekend this happens later and to a lesser extent, as people leave their houses to go to leisure activities, etc. On weekdays, lunch-time dips can be seen in node 1 , as people make a quick trip to home or to shops for example, where small spikes are visible in the other nodes. Another pattern worth noting in these graphs is that people return home later on Friday and especially Saturday night compared to Thursday, showing the high level of detail in the results that are obtained from the activities. Some travel variations between days are caused by different activities, while others may have to do with small 
deviations in departure times and where EVs are located based on random factors in the agent's choice model.

It is worth noting that Node 4, in Figure 6, clearly shows a much smaller volume of cars which can be explained by the fact that there is no housing near this node and the number of buildings, mostly leisure centres, is much smaller making it a less busy destination. However, it is interesting to see it has a much different profile than the other nodes, with people staying for long uninterrupted periods, especially on Saturday.

It should be stressed that the data presented in this section is output of the ABM, whereas other EV studies as indicated in Section II, often resort to composing this data as input without having the spatial elements clearly represented. By adding agents or changing their profiles in the model it is possible to create reliable forecasts of the behaviour of EV owners and hence the flexibility to forecast where and when to charge and how much. Consequently, the LEV, total battery energy used, and plug-in factors outputs provide the TCOPF with the required input data to optimise EV charging.

\section{Optimal Power Flow Model Results}

The TCOPF make the most of the ABM forecast on EV charging load flexibility resulting in ideal charging of EV fleets. Results contain valuable granular data that allows us to assess in-depth nodal charging taking place at each interval. Table IV compares basic network metrics that allow us to compare how low EV penetration impacts the local network assessed. The 'No EVs' scenario gives benchmark data on energy provided and network losses. Naturally, once EVs are included in the model the energy supplied by the slack bus and the losses in the network increase slightly due to the new burden EVs bring. Once EVs are considered, it is possible to calculate charging costs and carbon content that fuel these vehicles. In the second scenario charging costs are lower than in the third scenario, since the latter contains higher spot prices of electricity but even more significantly it is due to the $£ 60 / \mathrm{tCO}_{2}$ imposed for charging EVs with fossil fuelled power. However, the prowess of the TCOPF solver is seen when the third scenario charges its vehicle fleet with cleaner electricity.

Based on this case study and for sake of discussion, EVs in the UK would currently charge optimally with average grid carbon of $0.554 \mathrm{kgCO}_{2} / \mathrm{kWh}$, but if low carbon generation projects are fulfilled charging emissions could be reduced to about $0.393 \mathrm{kgCO}_{2} / \mathrm{kWh}$; thus having serious implications on $W 2 W$ efficiency studies - an exciting string of research [12].

Figures 7 and 8 show the power profile variations seen from the supply point once EVs are considered. Since penetration is low and there is a correlation between power demand and prices it can be expected that optimal charging will occur mostly in the valley of the static curves. The impartial TCOPF solver dispatches EVs differently in each scenario due to economic signals - this helps to highlight EV load flexibility.

Figures 9 and 10 depict where, when, and how much power the EV batteries take from the grid to satisfy its energy requirements. As it can be seen, the level of granularity is profound and this allows us to pin point spatial stochastic demands EVs bring along when penetration becomes prominent. Scenario 2 has more of its charging occurring in its residential nodes during the 3 days. Meanwhile, in scenario 3 even though the same pattern remains, there is more charging taking place in node 1 during the day while also the capacities being drawn from residential nodes are slightly different due to economic signals. No charging takes place in node 4 because while EVs are plugged it is not that attractive to do so - making it clear that if the solver forecasts it will be cheaper to charge at a later moment of the day it will do so.

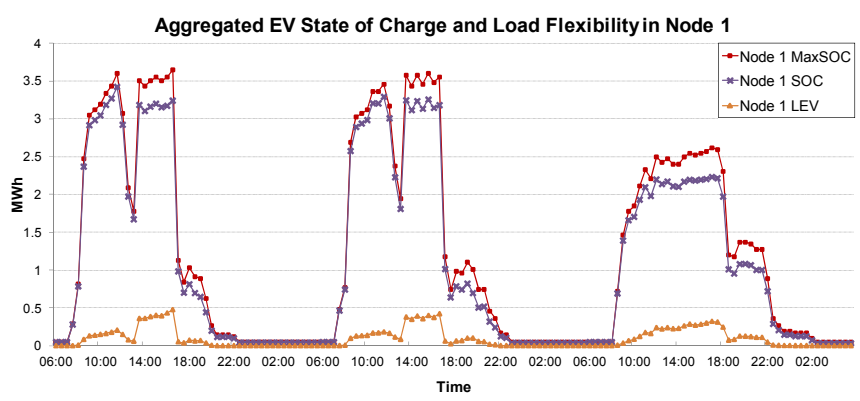

Fig. 3 - Node 1 describes how commercial areas can monitor EV influence.

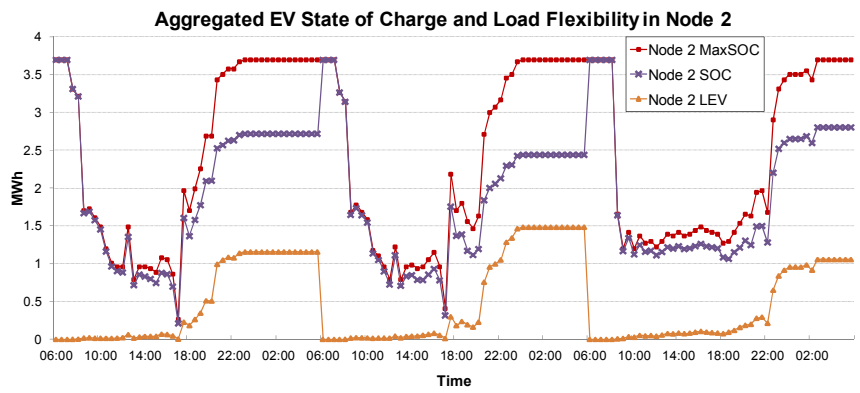

Fig. 4 - Node 2 describes how residential areas can monitor EV influence.

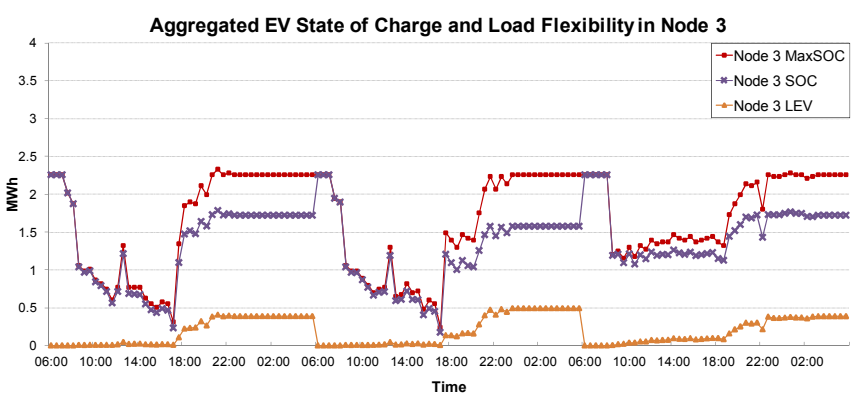

Fig. 5 - Node 3 describes how residential areas can monitor EV influence.

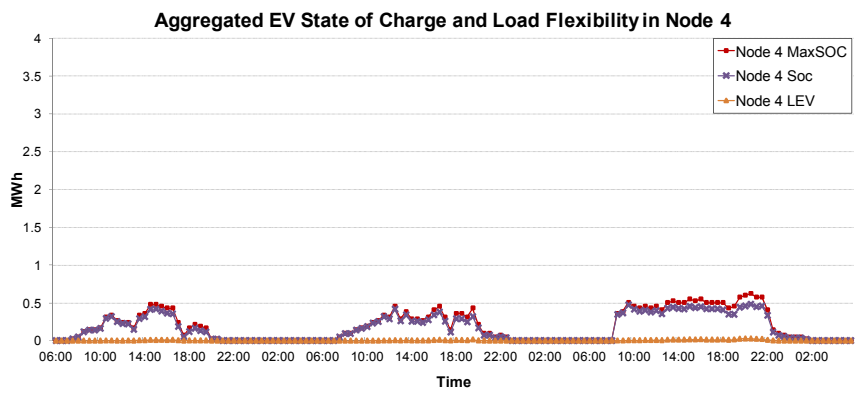

Fig. 6 - Node 4 describes how residential areas can monitor EV influence. 
As the result description suggests, the variations on input data and modelling assumptions can make model output vary significantly. Indeed, each case study needs to be carefully formulated. However, the ability to formulate and calculate complex problems such as EV mobility and optimal charging is a strong asset that integrated modelling brings to the forefront of power system studies.

TABLE IV

ELECTRIC NETWORK AND EV RESULTS

\begin{tabular}{|c|c|c|c|c|}
\hline $\begin{array}{c}\text { Case } \\
\text { Scenario }\end{array}$ & $\begin{array}{c}\text { Losses } \\
(\mathbf{M W h})\end{array}$ & $\begin{array}{c}\text { Slack } \\
(\mathbf{M W h})\end{array}$ & $\begin{array}{c}\text { Carbon of } \boldsymbol{E} \boldsymbol{V} \\
\text { Charging }\left(\boldsymbol{t} \boldsymbol{C O}_{2}\right)\end{array}$ & $\begin{array}{c}\text { Cost of } \boldsymbol{E} \boldsymbol{V} \\
\text { Charging (f) }\end{array}$ \\
\hline 1- No EVs & 14.63 & 507.86 & 0.00 & 0.00 \\
\hline 2- Present & 14.82 & 512.97 & 2.72 & 244.01 \\
\hline 3- Future & 14.82 & 512.97 & 1.93 & 435.40 \\
\hline
\end{tabular}

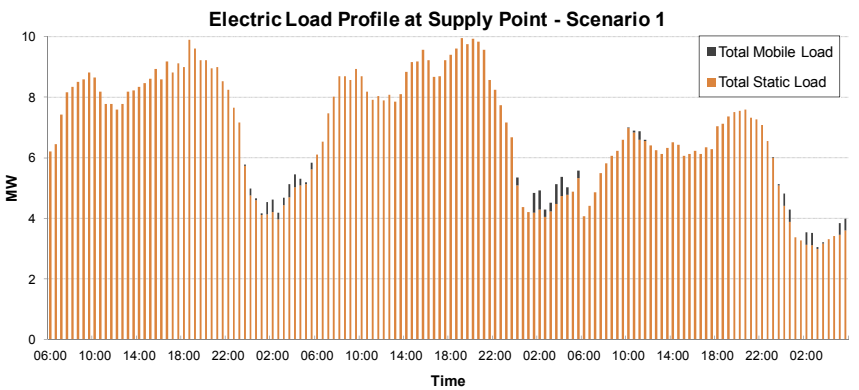

Fig. 7 - Illustrates optimal EV charging in scenario 1 - making a clear distinction between conventional (static) and EV (mobile) load.

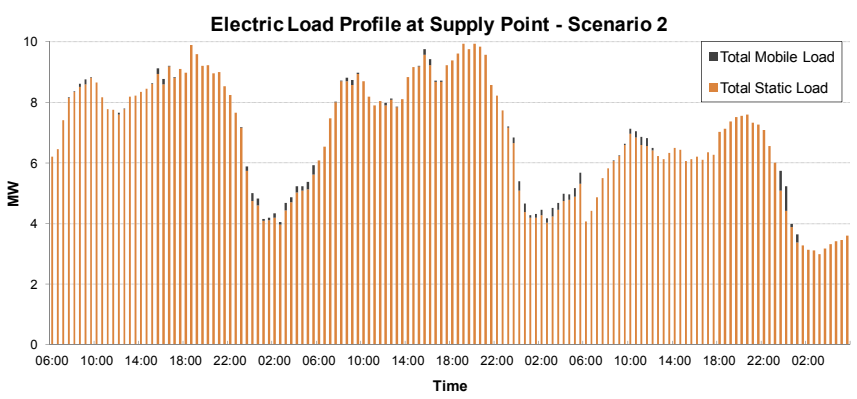

Fig. 8 - Illustrates optimal EV charging in scenario 2 - making a clear distinction between conventional (static) and EV (mobile) load.

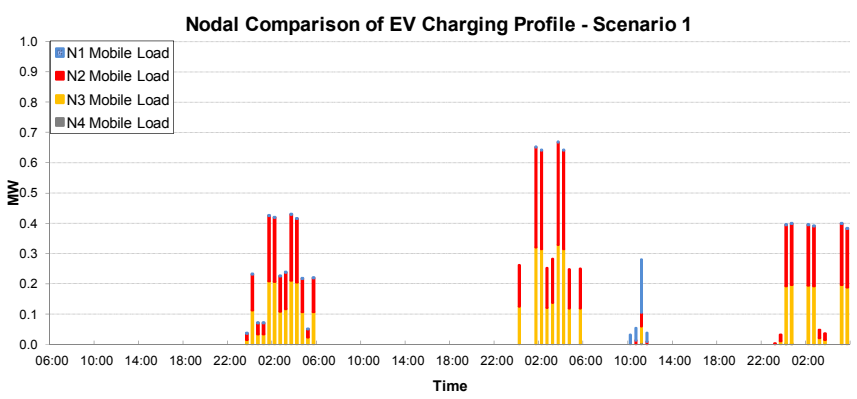

Fig. 9 - Nodal charging of EVs in scenario 1 - most of the charging takes place at night time in residential nodes, some charging occurs in node 1 on Saturday morning.

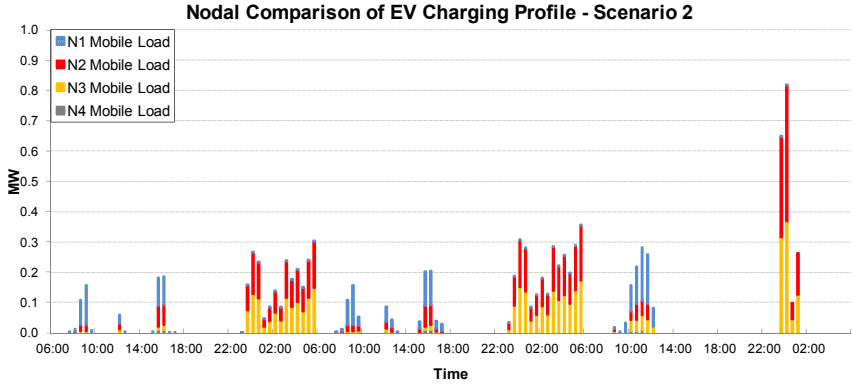

Fig. 10 - Nodal charging of EVs in scenario 2 -the charging is more distributed here although residential charging continues to dominate.

\section{CONCLUSIONS AND FURTHER WORK}

The ability to determine optimal charging profiles of EVs is paramount in developing an efficient and reliable smartgrid. This paper has attempted to merge transport related modelling concepts from EV travel with traditional optimal power flow issues in order to identify ideal EV charging strategies that enhance power network performance.

A thorough literature review suggests that power system engineers have struggled to tackle this problem properly due to the lack of modelling formulations that can represent the travel behaviour of vehicles and the logging of its activities. By using an ABM to calculate journeys and energy demands of EV owners it is shown the possibility exists to draw key information that serves utilities to forecast the moments of the day when it can exploit load flexibility of EVs. In this manner, the temporal and spatial conundrum of these mobile loads can be addressed by power system engineers.

After each daily travel profile from the agents has been calculated, the aggregated spatial data of where EVs are located as well as their battery SOC can be introduced into the TCOPF model. The optimal power flow considers the energy it needs to supply, the EV load flexibility constraints in each node, plus the price signals from various sources to determine the best moments to charge the units. Results from the case study show a high level of detail and variability in EV charging when a present-day carbon fuel mix is compared to one with lower carbon intensity. The TCOPF solver offers valuable granular data describing the ideal charging of EVs a novel form of result from the author's perspective. Due to the objective cost function in place, results indicate that optimal EV charging can improve the use of existent power infrastructure. Scenarios to model are endless once the integration of transport and power sector studies is possible.

Further work in this field of research can take many directions, however key prominence should be given first to strengthening the modelling principles used here in order to enhance the quality and depth of results an integrated model can offer. The authors suggest incentivised EV charging via power markets and its corresponding commercial framework can now be better analysed once an analytical tools such as the one presented here are more prominent. Likewise, operational and design network studies can be developed once EV load growth forecasting can be determined more effectively. 


\section{REFERENCES}

[1] Grainger, J. and Stevenson, W. Power System Analysis, McGraw-Hill, New York, 1994, ISBN 0070612935.

[2] Kundur. P., Balu, N. and Lauby, M. Power System Stability and Control, McGraw-Hill, New York, 1994, ISBN 007035958X.

[3] Acha, S.; Green, T.C.; Shah, N.; , "Techno-economical tradeoffs from embedded technologies with storage capabilities on electric and gas distribution networks," Power and Energy Society General Meeting, 2010 IEEE, pp.1-8, 25-29 July 2010

[4] International Energy Agency, "Technology Roadmap - Electric and plug-in hybrid electric vehicles" (updated June 2011). [Online]. Available: http://www.iea.org/roadmaps/plug_in_electric_vehicles.asp [Accessed: 7 November 2011].

[5] AEA Group, "Market outlook to 2022 for battery electric vehicles and plug-in hybrid electric vehicles" (updated June 2009). [Online]. http://www.aeat.com/cms/a-market-outlook-to-2022-for-battery-electricand-plug-in-hybrid-electric-vehicles/[Accessed: 21 Nov 2011].

[6] Robert C. Green II, Lingfeng Wang, Mansoor Alam, The impact of plugin hybrid electric vehicles on distribution networks: A review and outlook, Renewable and Sustainable Energy Reviews, Volume 15, Issue 1, January 2011, Pages 544-553, ISSN 1364-0321.

[7] Bessa, R. J. and Matos, M. A., Economic and technical management of an aggregation agent for electric vehicles: a literature survey. European Transactions on Electrical Power, 2011

[8] Acha, S., van Dam, K.H., Keirstead, J. and Shah, N., "Integrated modelling of agent-based electric vehicles into optimal power flow studies," Proceedings of CIRED 2011. pp.1-4, 6-8 June 2011.

[9] Keenan Valentine, William G. Temple, K. Max Zhang, Intelligent electric vehicle charging: Rethinking the valley-fill, Journal of Power Sources, Volume 196, Issue 24, 15 December 2011, Pages 10717-107

[10] Verzijlbergh, R.A.; Ilic, M.D.; Lukszo, Z.; , "The role of electric vehicles on a green island," North American Power Symposium (NAPS), 2011, pp.1-7, 4-6 Aug. 2011

[11] Sortomme, E.; Hindi, M.M.; MacPherson, S.D.J.; Venkata, S.S "Coordinated Charging of Plug-In Hybrid Electric Vehicles to Minimize Distribution System Losses," Smart Grid, IEEE Tran..2(1), pp.198-205, March 2011

[12] Acha, S.; Green, T.C.; Shah, N., "Optimal charging strategies of electric vehicles in the UK power market," Innovative Smart Grid Technologies (ISGT), 2011 IEEE PES, pp.1-8, 17-19 Jan. 2011

[13] Kristoffersen, T.K., Capion, K. and Meibom, P. Optimal charging of electric drive vehicles in a market environment, Applied Energy, Volume 88, Issue 5, May 2011, Pages 1940-1948, ISSN 0306-2619

[14] Kang, J. E. and Recker, W.W. An activity-based assessment of the potential impacts of plug-in hybrid electric vehicles on energy and emissions using 1-day travel data, Transp. Research Part D: Transport and Environment, Volume 14, Issue 8, December 2009, Pages 541-556

[15] Galus, M., Dobler, C., Balmer, M., Andersson, G., Axhausen, K., 2009. Plug-in hybrid electric vehicles and smart grid: investigations based on a micro-simulation. In: Conference of the International Association for Travel Behaviour Research, IABTR, Jaipur, India

[16] Matthias D. Galus, R. A. Waraich, Göran Andersson, Predictive, distributed, hierarchical charging control of PHEVs in the distribution system of a large urban area incorporating a multi agent transportation simulation, Presented at the 17th Power Systems Computation Conference, Stockholm, Sweden, 2011

[17] Eppstein, M.J., Grover, D.H., Marshall, J.S. and Rizzo, D.M. An agentbased model to study market penetration of plug-in hybrid electric vehicles, Energy Policy 39(6), June 2011, Pages 3789-3802

[18] Sweda, T.; Klabjan, D.; , "An agent-based decision support system for electric vehicle charging infrastructure deployment," Vehicle Power and Propulsion Conference (VPPC), 2011 IEEE , pp.1-5, 6-9 Sept. 2011

[19] J. K. Kok, C. J. Warmer, and I. G. Kamphuis. 2005. PowerMatcher: multiagent control in the electricity infrastructure. In Proceedings of the fourth international joint conference on Autonomous agents and multiagent systems (AAMAS '05). ACM, New York, NY, USA, 75-82

[20] Bliek, F.; van den Noort, A.; Roossien, B.; Kamphuis, R.; de Wit, J.; van der Velde, J.; Eijgelaar, M.; , "PowerMatching City, a living lab smart grid demonstration," Innovative Smart Grid Technologies Conference Europe (ISGT Europe), 2010 IEEE PES

[21] Vytelingum, P., Voice, T., Ramchurn, S., Rogers, A. and Jennings, N. (2011) Theoretical and Practical Foundations of Agent-Based MicroStorage in the Smart Grid. J. of Artificial Intelligence Res.. (In Press)
[22] Bri-Mathias S. Hodge, Shisheng Huang, Aviral Shukla, Joseph F. Pekny, Gintaras V. Reklaitis The Effects of Vehicle-to-Grid Systems on Wind Power Integration in California, Computer Aided Chemical Engineering, Volume 28, 2010 , Pages 1039-1044

[23] Vandael, S., De Craemer, K., Boucké, N., Holvoet, T. Deconinck, G. Decentralized coordination of plug-in hybrid vehicles for imbalance reduction in a Smart Grid, Proc. of the 10th Intl. Conf. on Autonomous Agents and Multiagent Systems (AAMAS 2011) pages:803-810

[24] Malleson, N. (2008) "RepastCity - A demo virtual city" [online] http://portal.ncess.ac.uk/wiki/site/mass/repastcity.html (1 May 2011)

[25] Acha, S., PhD Thesis (2010), "Impacts of Embedded Technologies on Optimal Operation of Energy Service Networks", Imperial College London, UK.

[26] Acha, S.; Green, T.C.; Shah, N. "Effects of optimised plug-in hybrid vehicle charging strategies on electric distribution network losses," Transmission and Distribution Conference and Exposition, 2010 IEEE PES ,pp.1-6, 19-22 April 2010

[27] Gproms software. [Online]. Available: http://www.psenterprise.com

[28] UKGDS, "United Kingdom Generic Distribution System", [Online]. Available: monaco.eee.strath.ac.uk/ukgds/, accessed on July 12, 2009.

\section{BIOGRAPHIES}

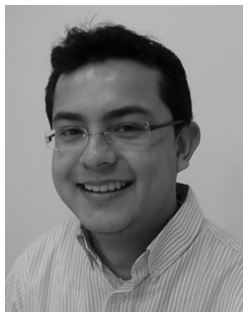

Salvador Acha received a B.Sc. (Eng.) degree in Electronics and Communications Engineering from Monterrey Tech, in Mexico, in 2003. After working in the private sector he joined Imperial College London in 2006 where he obtained a Ph.D. degree in Electrical Engineering in 2010 for his thesis on optimally integrating DERs into infrastructures. Currently he is a Research Associate at Imperial with interests including smart-grids, roll out of PHEV, and optimisation of distributed energy sources.

Koen H. van Dam holds an MSc degree in Artificial Intelligence from the

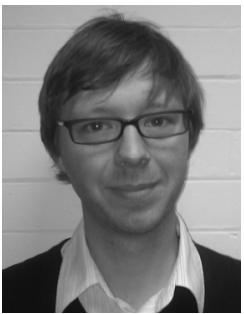
Vrije Universiteit in Amsterdam and a $\mathrm{PhD}$ degree from the Delft University of Technology awarded in 2009 for his thesis on modelling socio-technical infrastructure systems. Since 2011 he is a Research Associate at Imperial working on the Digital City Exchange project. Previously he worked as post-doc at TU Delft and as visitor at NUS and Imperial. His research interests include urban energy areas, and ABM modelling.

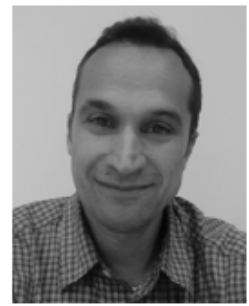

Nilay Shah obtained his Ph.D. in Chemical Engineering from Imperial College London in 1992. After a period at Shell UK, he joined the academic staff of Imperial College. Since 2001 he is Professor of Process Systems Engineering and he is the deputy director of CPSE, co-director of the BP Urban Energy System project and a Fellow of the Institution of Chemical Engineers. His research interests include application of mathematical and systems engineering techniques to analyse and optimise energy systems. 